\title{
Diffuse pleural thickening: cases of pseudomesotheliomatous adenocarcinoma and pleural tuberculosis
} Amrith B.P. ${ }^{a}$, Animesh Ray ${ }^{\mathrm{a}}$, Aanchal Kakkar ${ }^{\mathrm{b}}$, Sanjeev Sinha ${ }^{\mathrm{a}}$

Diffuse pleural thickening is a common cause of diagnostic dilemma. We report two cases of pleural thickening that presented with similar clinical and radiological picture, thus clinching a diagnosis hinged on histopathology. In the first case, the histopatholgy and immunohistochemistry was suggestive of adneocarcinoma, thus making the diagnosis of pseudomesotheliomatous adenocarcinoma. In the second case, the histopathology was suggestive of tubercular etiology, which also is a rare presentation of active pleural tuberculosis. These cases highlight the importance of histopathological examination in establishing the etiology in cases of diffuse pleural thickening.

Egypt J Bronchol 2018 12:358-362

(C) 2018 Egyptian Journal of Bronchology

\section{Introduction}

Pleural thickening can be diffuse or focal, which may be of benign or malignant etiology. Diffuse pleural thickening is defined as pleural thickening of more than $5 \mathrm{~mm}$ with combined area of involvement more than $50 \%$ if unilateral, or more than $25 \%$ if bilateral [1]. Owing to multiple etiologies of pleural thickening, it may be difficult to identify the exact cause by imaging alone.

The stated two cases presented with similar clinical and radiological picture, but the histopathological examination revealed adenocarcinoma in one case and tuberculosis in the other. This highlights the importance of histopathologic correlation by the help of suitable technique in cases of diffuse pleural thickening.

\section{Case 1}

A 31-year-old female patient presented to the OPD with history of left-sided chest pain and progressive dyspnea for 1 year. She also complained of low-grade intermittent fever, weight loss and loss of appetite for the same duration. She was a homemaker, without any significant occupational exposure, or history of smoking. She was treated with antitubercular therapy for past 4 months from an outside hospital, based on exudative pleural fluid. However, there was no microbiological evidence for the same. There was no symptomatic relief while on treatment. On respiratory system examination, she had decreased intensity of breath sounds on the left side.

Her chest radiograph showed left-sided diffuse pleural thickening. Computed tomography (CT) of the chest
Egyptian Journal of Bronchology 2018 12:358-362

Keywords: diffuse pleural thickening, pleural tuberculosis, pseudomesotheliomatous adenocarcinoma

Departments of, aMedicine, ${ }^{b}$ Pathology, All India Institute of Medical Sciences, New Delhi, India

Correspondence to Animesh Ray, DM, Department of Medicine, All India Institute of Medical Sciences, New Delhi 110029, India Tel: +91956009 3190;

e-mail: doctoranimeshray@gmail.com

Received 22 December 2017 Accepted 2 July 2018

showed diffuse, nodular thickening $(>1 \mathrm{~cm})$ of the left pleura involving costal, mediastinal, and diaphragmatic surfaces (Fig. 1). Positron emission tomography scan showed metabolically active left pleural thickening (Fig. 2). The overall picture was suggestive of a pleural malignancy. CT-guided biopsy from the pleural thickening showed cores of fibrocollagenous tissue infiltrated by a tumor with cells arranged in cords, and in glandular pattern. Tumor cells lining the glands were cuboidal to columnar, with abundant pale, eosinophilic cytoplasm showing vacuoles at places, and basal, hyperchromatic nuclei. On immunohistochemistry, the tumor cells revealed positivity for pancytokeratin, epithelial membrane antigen, and thyroid transcription factor 1 (TTF-1), whereas they were negative for calretinin and D2-40 (Fig. 3). Based on these features, a diagnosis of lung adenocarcinoma was rendered. The clinicoradiological presentation was consistent with pseudomesotheliomatous adenocarcinoma, and she was managed accordingly.

\section{Case 2}

A 26-year-old female patient presented with history of intermittent low-grade fever for 6 months. It was associated with dull aching right-sided chest pain. She was a nonsmoker, and there was no significant occupational exposure. Examination of the chest revealed reduced expansion of the right hemithorax.

This is an open access journal, and articles are distributed under the terms of the Creative Commons Attribution-NonCommercial-ShareAlike 4.0 License, which allows others to remix, tweak, and build upon the work non-commercially, as long as appropriate credit is given and the new creations are licensed under the identical terms. 
The evaluation of her chest radiograph showed blunting of the right costophrenic angle with normal lung parenchyma. Ultrasonography (USG) chest showed

Figure 1

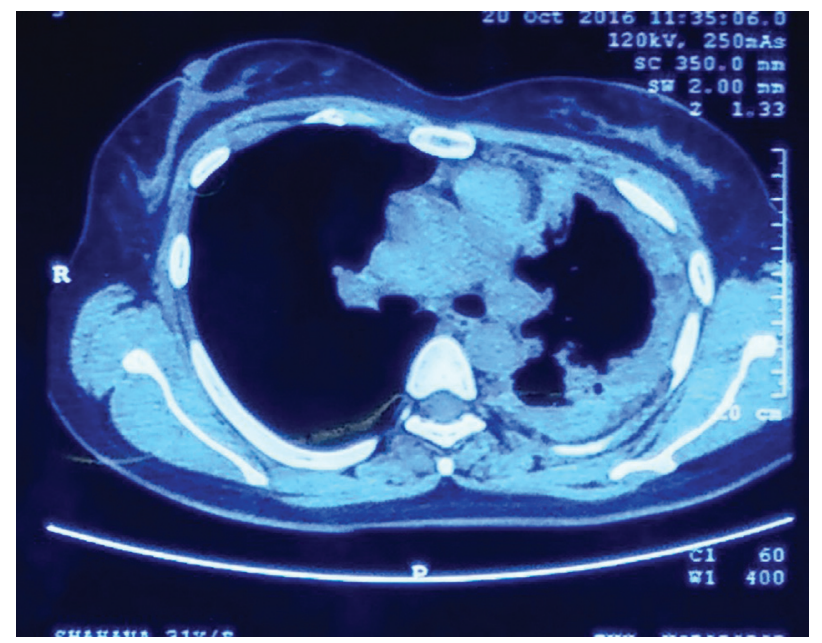

Computed tomography of the chest showing diffuse, nodular thickening of the left pleura. thickening of the right pleura without any fluid collection. Contrast-enhanced CT (Fig. 4) of the chest was done to further characterize the pleural thickening, which showed diffuse nodular thickening

\section{Figure 2}

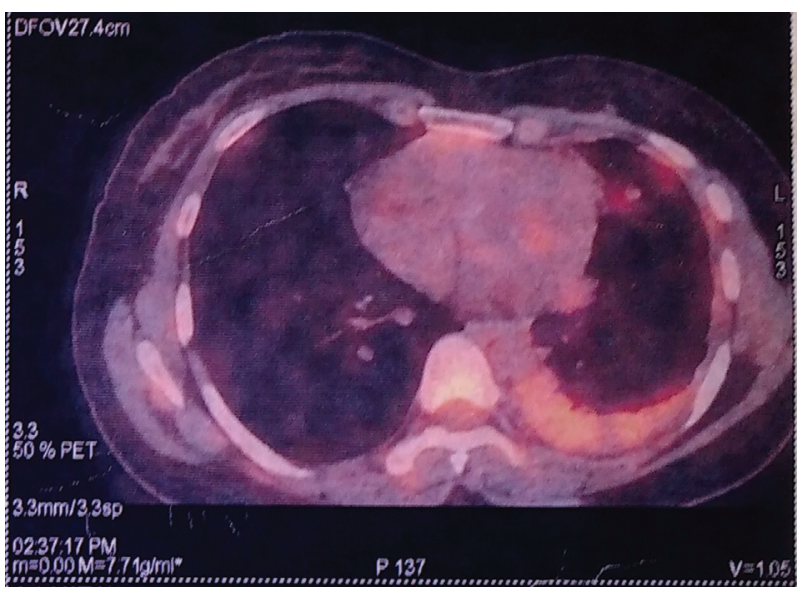

Positron emission tomography scan showing metabolically active left pleural thickening.

Figure 3

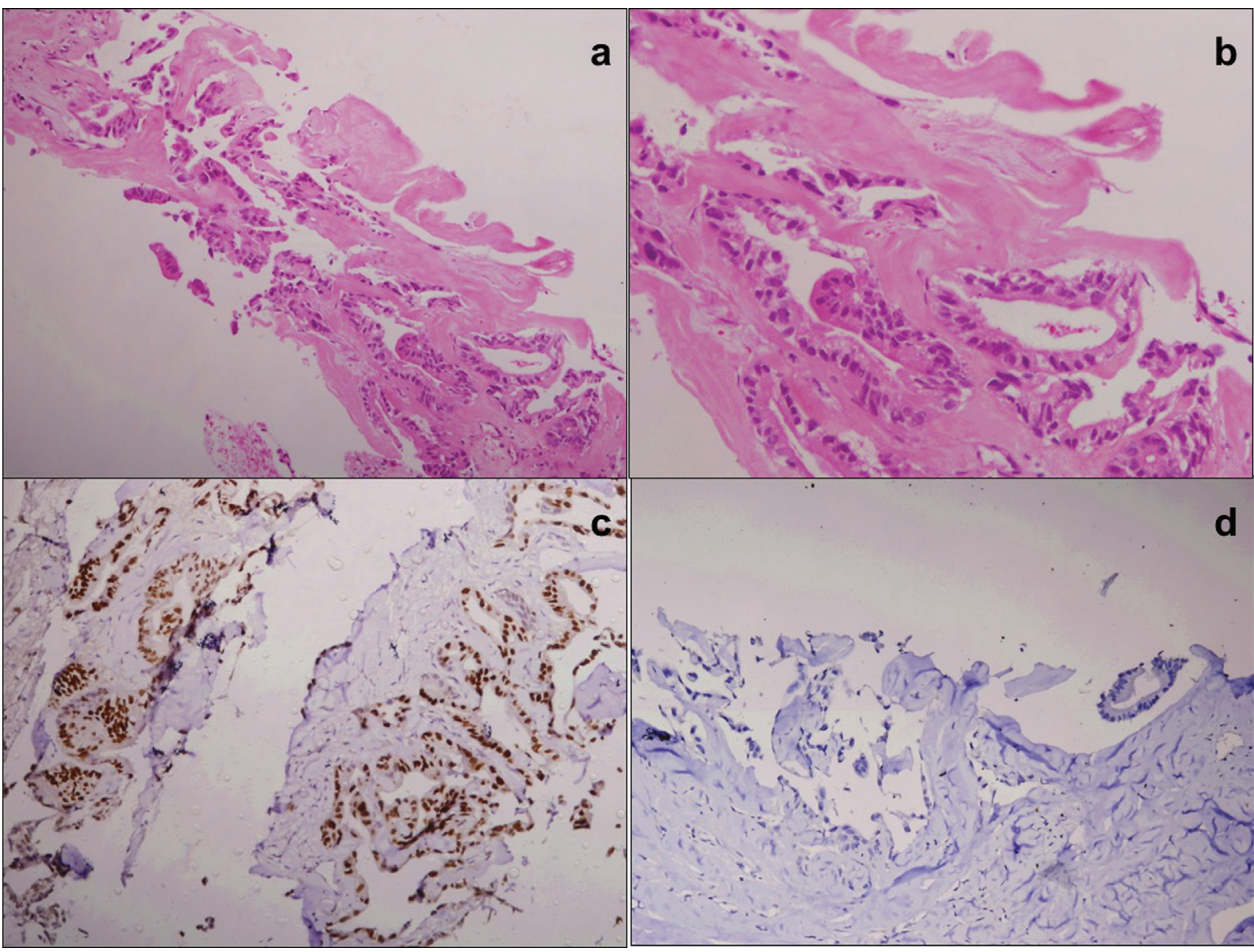

Histopathological sections showing cores of fibrocollagenous tissue infiltrated by a tumor with cells arranged in cords, and in glandular pattern Tumor cells lining the glands were cuboidal to columnar, with abundant pale, eosinophilic cytoplasm showing vacuoles at places, and basal, hyperchromatic nuclei. On immunohistochemistry, the tumor cells revealed positivity for pancytokeratin, epithelial membrane antigen, and TTF1 , whereas they were negative for calretinin and D2-40. 
$(>1 \mathrm{~cm})$ of the right pleura involving mediastinal, costal and diaphragmatic surfaces, with mild volume loss, raising the possibility of pleural metastasis or mesothelioma. A biopsy was performed under CT guidance, which showed necrotizing granulomatous inflammation suggestive of tubercular etiology. She was started on antitubercular therapy and closely followed up. Mycobacterial culture of the pleural tissue was also positive for drug-sensitive tuberculosis. The patient responded to the therapy with resolution of her complaints. Figures 5 and 6 show the pretreatment

\section{Figure 4}

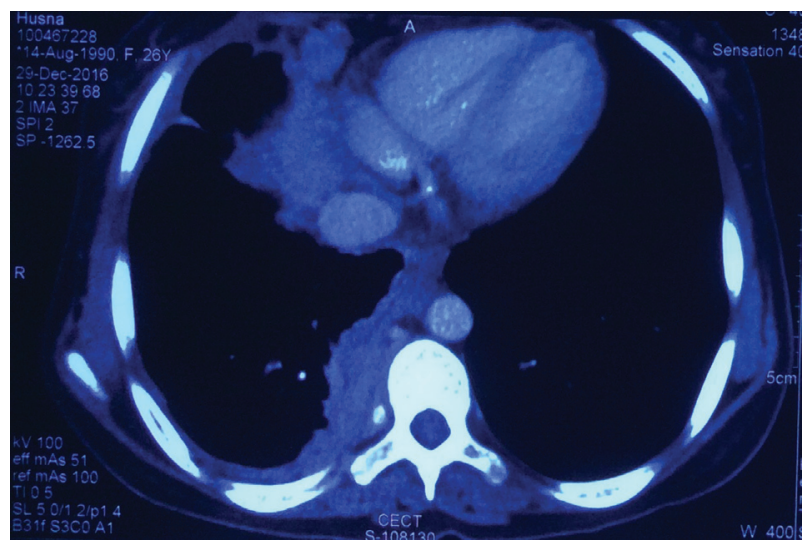

Computed tomography chest showing diffuse nodular thickening of the right pleura.

\section{Figure 5}

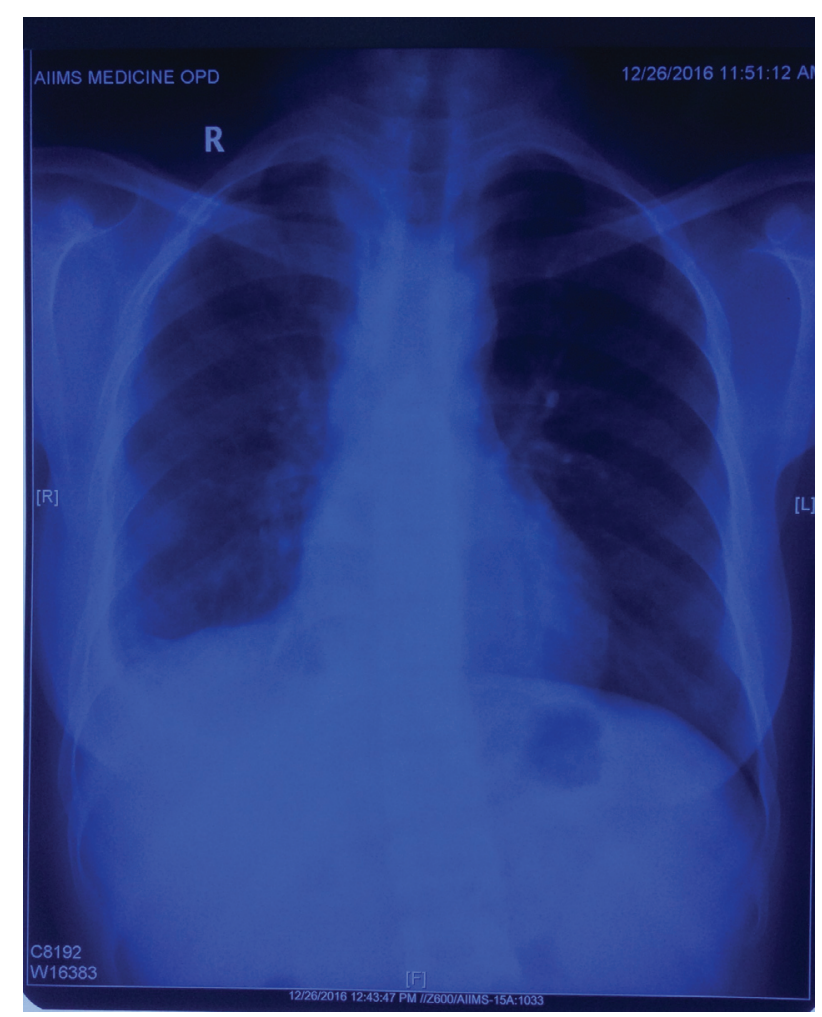

Chest radiography pretreatment. and follow-up radiography of the patient after 4 months of treatment.

\section{Discussion}

Pleural plaques, pleural fibroma, and metastasis are important causes of focal pleural thickening. Diffuse pleural thickening is usually associated with occupational exposure (asbestosis) and may occur secondary to empyema (tuberculosis) or inflammation related to connective tissue diseases. Pleural mesothelioma, lymphoma, and pleural metastasis, especially from adenocarcinoma of lung, can present as focal or diffuse pleural thickening. Various causes for diffuse pleural thickening are listed in the Table 1 [2].

On plain radiography, the pleural thickening will have poorly defined margins and need a further imaging modality to confirm the findings. USG is a poor modality to characterize the pleural thickening [3]. It can be used to assess the fluid collection in the pleural cavity. Though CT scan with contrast enhancement might give some clues to the underlying etiology, a histopathological examination is vital in establishing the definite etiology in doubtful cases. CT features suggestive of malignant etiology are pleural thickening of greater than $1 \mathrm{~cm}$, nodularity and mediastinal pleural involvement [4]. Biopsy under CT/ USG guidance is usually performed by radiologists to obtain a tissue diagnosis. Blind biopsies are an obsolete procedure now, and they carry significant risk of

\section{Figure 6}

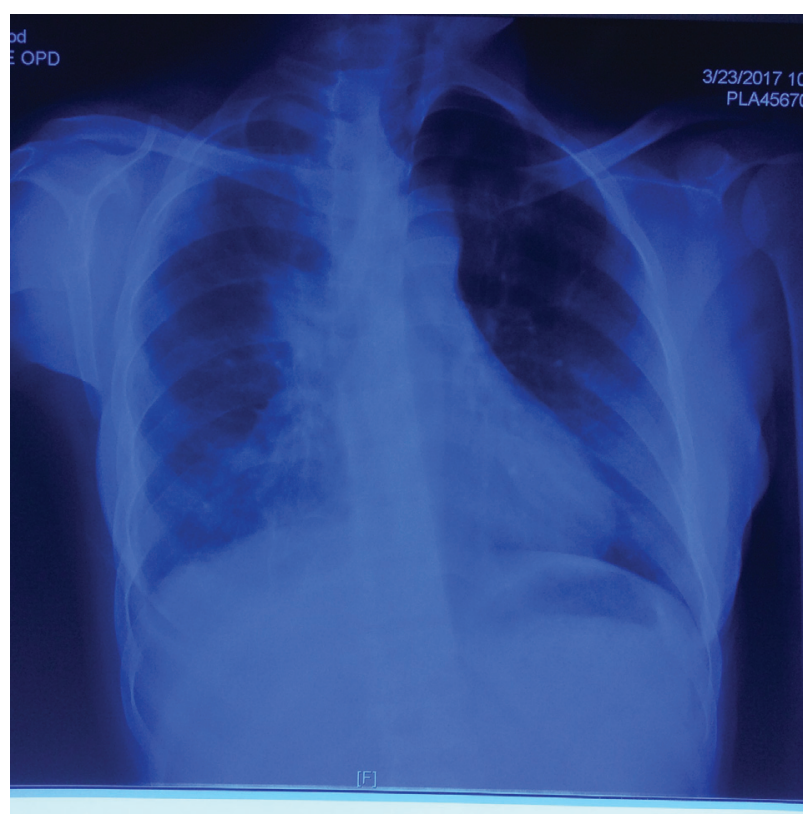

Chest radiography after 4 months of antitubercular therapy. 
complications. Thoracoscopy has the additional benefit of visualizing the pleural surfaces, and it may be considered if the required expertise is available [5].

Pseudomesotheliomatous adenocarcinoma refers to tumors whose radiological or thoracoscopic picture resembles that of pleural mesothelioma but histologic and immunohistochemical features are those of a tumor other than mesothelioma, usually of a peripheral lung adenocarcinoma. Although adenocarcinomas can closely mimic epithelioid mesotheliomas, particularly in small biopsies, the use of an appropriate immunohistochemical panel helps to resolve the diagnostic dilemma, as in the present case. In cases with overlapping immunohistochemical features, electron microscopy serves as a valuable diagnostic adjunct. Pathological features differentiating pseudomesotheliomatous adenocarcinoma from an adenocarcinoma of lung are presented in the Table 2 [6].

Pseudomesotheliomatous adenocarcinoma is a rare presentation of lung carcinoma, and it has been rarely reported from the Indian subcontinent. The largest case series of pseudomesotheliomatous neoplasms

\section{Table 1 Causes of diffuse pleural thickening}

Infections - empyema and tuberculosis
Occupational exposures - asbestosis and silicosis
Pleural hemorrhage
Neoplasms - mesothelioma, metastasis, and primary pleural
lymphoma
Post radiation
Pulmonary embolism
Erdheim-chester disease
Diffuse pulmonary lymphangiomatosis

Table 2 Histopathology and immunohistochemistry features to differentiate mesothelioma and pseudomesotheliomatous adenocarcinoma.

\begin{tabular}{lcc}
\hline & Mesothelioma & $\begin{array}{c}\text { Pseudomesotheliomatous } \\
\text { adenocarcinoma }\end{array}$ \\
\hline $\begin{array}{l}\text { Histology } \\
\text { PAS }\end{array}$ & Negative & Positive \\
Mucicarmine & Negative & Positive \\
Immunohistochemistry & Negative & Positive \\
TTF-1 & Positive & Negative \\
Calretinin & Negative & Positive (75\%) \\
CEA & Negative & Positive \\
Leu M1 & Negative & Positive \\
Ber EP4 & Negative & Positive \\
B72.3 & Positive & Negative \\
Thrombomodulin & & Short \\
Electron microscopy & & Long \\
Microvilli & Lodid
\end{tabular}

CEA, carcinoembryonic antigen; PAS, periodic acid schiff. was published by Hammar and Dodson [7] in 2015 from their experience over 32 years. Of 196 patients, 183 patients had a primary in the lung, two patients had primary in the peritoneum and 11 were metastatic from other sites. However, this type of presentation has also been reported from cancers other than adenocarcinomas. Small-cell neuroendocrine tumors of the lung can have diffuse pleural seeding leading to a mesotheliomatous appearance [8].

Residual pleural thickening is a common sequelae of pleural tuberculosis. Approximately $50 \%$ of the patients show residual pleural thickening when their therapy is completed [9]. However, pleural tuberculosis presenting as isolated pleural thickening without pleural effusion is rare, and needs a high index of suspicion to diagnose.

\section{Conclusion}

The aforementioned two cases of pleural thickening presented with similar clinical and radiological picture; however, the final diagnoses were diametrically different. In the first case, histopathology and immunohistochemistry was suggestive of adenocarcinoma - a rare entity of pseudo- mesotheliomatous adenocarcinoma. The second case was proved to be owing to tuberculosis, which also is a rare presentation of active pleural tuberculosis. In both of these cases, CT-guided biopsy was crucial in establishing the definitive diagnosis. These cases highlight the importance of histopathological examination while approaching a case of pleural thickening, as short of it, clinical and imaging findings may not clinch the correct diagnosis.

\section{Financial support and sponsorship}

Nil.

\section{Conflicts of interest}

There are no conflicts of interest.

\section{References}

1 Sureka B, Mittal M, Mittal A, Sinha M, Thukral B. Radiological review of pleural tumors. Indian J Radiol Imaging 2013; 23:313.

2 Walker CM, Takasugi JE, Chung JH, Reddy GP, Done SL, Pipavath SN, et al. Tumorlike conditions of the pleura. Radiographics 2012; 32:971-985

3 Chen S-W., Zhang M-Y., Liu J. Application of lung ultrasonography in the diagnosis of childhood lung diseases. Chin Med J (Engl) 2015; 128:2672-2678.

4 Hierholzer J, Luo L, Bittner RC, Stroszczynski C, Schroder RJ, Dorow P, et al. MRI and CT in the differential diagnosis of pleural disease. Chest 2000 ; 118:604-609. 
5 Dixon G, de Fonseka D, Maskell N. Pleural controversies: image guided biopsy vs. thoracoscopy for undiagnosed pleural effusions? J Thorac Dis 2015; 7:1041-1051.

6 Pardo J, Torres W, Martinez-Peñuela A, Panizo A, de Alava E, García JL. Pseudomesotheliomatous carcinoma of the lung with a distinct morphology, immunohistochemistry, and comparative genomic hybridization profile. Ann Diagn Pathol 2007; 11:241-251.

7 Hammar SP, Dodson RF. Pseudomesotheliomatous lung carcinoma: a pathological assessment of selected cases including the work history and presence of biomarkers suggesting it is a cancer caused by asbestos. Pathol Discov 2015; 3:6.

8 Exarchos GD, Attanoos RL. Pseudomesotheliomatous small-cell neuroendocrine carcinoma of the lung with calretinin expression. Histopathology 2015; 66:895-896.

9 Barbas CSV, Cukier A, de Varvalho CRR, Filho JVB, Light RW. The relationship between pleural fluid findings and the development of pleural thickening in patients with pleural tuberculosis. Chest 1991; 100:1264-1267. 